\title{
DISEÑO DE UNA ONTOLOGÍA PARA AGENTES QUE MONITOREAN MEDICIONES DE SENSORES
}

\section{DESIGN OF AN ONTOLOGY TO AGENTS THAT MONITORING MEASUREMENTS OF SENSORS}

\author{
Ing. Angela Patricia Hernández Cruz, PhD. Carlos Arturo Parra Ortega. \\ MSc. Luis Armando Portilla Granados.
}

Universidad de Pamplona, Programa de Ingeniería de Sistemas. Ciudadela Universitaria. Pamplona, Norte de Santander, Colombia. Tel.: (+577) 568 5303, Fax: (+577) 5685303, Ext. 273.

E-mail: apjonat@hotmail.com, \{carapa, laporti \}@unipamplona.edu.co

\begin{abstract}
Resumen: En este artículo se propone el uso de sistemas multi-agente y ontologías para solucionar el problema de monitoreo de procesos heterogéneos cuyos datos son adquiridos mediante sensores. La representación de información mediante ontologías permite un mejor control de proceso, considerando que el monitoreo es un aspecto fundamental en la automatización de procesos industriales. Para probar el diseño de la ontología se aplicó al problema de controlar un proceso industrial, simulando el proceso mediante un sistema multi-agente cuyo entorno está basado en una plataforma hecha en lenguaje de programación Java. El resultado de la simulación demuestra que la ontología diseñada facilita las actividades asociadas al monitoreo de diversas variables, y al control de procesos.
\end{abstract}

Palabras clave: Agentes, Sistemas multi-agente, adquisición de datos, ontologías.

\begin{abstract}
In this paper are proposed the use of multi-agent systems and ontologies in order to solve the problem of monitoring heterogeneous processes whose data are adquired by sensors. Representing information using ontology allows a better control and supervision, considering that monitoring is a fundamental issue in automating industrial processes. To probe the design of the ontology this was applied to control an industrial process, simulating it by a multi-agent system which is a java-based environment. The results of simulations show that the ontology designed makes easy the activities related to monitoring some variables, and process controlling.
\end{abstract}

Keywords: Agents, multi-agent systems, data acquisition, ontologies.

\section{INTRODUCCIÓN}

El monitoreo de variables en un contexto de automatización es un tópico de gran relevancia, puesto que es fundamental en la integración de sistemas y control de procesos.

En este trabajo se propone el uso de ontologías y sistemas multi-agente para resolver los problemas asociados al monitoreo de las lecturas de los sensores en procesos heterogéneos, permitiendo así una mejor y oportuna detección de eventos que lleven a tomar decisiones de control del proceso que se esté monitoreando.

Algunos trabajos similares en el área se han realizado, como la implementación de agentes en la arquitectura de automatización ANEMONA (Giret, 
2005) aunque no usa detección de eventos ni ontologías. También se ha propuesto un esquema de automatización de procesos con detección de eventos y agentes (Parra et al, 2008) con paso de mensajes simples.

Una ontología para automatización y control basada en el paradigma multi-agente y holónico se ha planteado en para obtener supervisión inteligente de procesos (Quintero 2010), aunque no usan la ontología para detección de eventos. También existen aplicaciones de agentes en detección de eventos de intrusiones en redes (Herrera y Parra, 2013).

El artículo está organizado en tres momentos investigativos, uno, con una recopilación teórica sobre los fundamentos asociados a la automatización, el segundo, detalla el proceso del diseño de la ontología y finalmente su implementación con una aplicación que simula un proceso de secado de café, donde se estudian los resultados y se generan las principales conclusiones.

\section{MONITOREO DE VARIABLES MEDIANTE SENSORES}

La automatización de procesos industriales se compone de varias fases iterativas, las cuales son el monitoreo de variables, la detección de eventos, la decisión y ejecución de medidas de control.

El monitoreo de variables físicas es un aspecto crítico, ya que dependiendo del valor que tomen las variables se puede establecer la ocurrencia de un evento determinado, que lleve a una acción de control.

La figura 1 muestra el esquema de funcionamiento de un proceso automatizado, donde las variables del proceso se miden a través de sensores, y sus lecturas se transmiten por medio de una red industrial a aplicaciones corporativas, objetos que llevan a cabo tareas o sistemas de control (Chacón, 2004).

Algunas acciones de los sistemas de control pasan al actuador, quien las aplica directamente sobre el proceso industrial a ser automatizado.

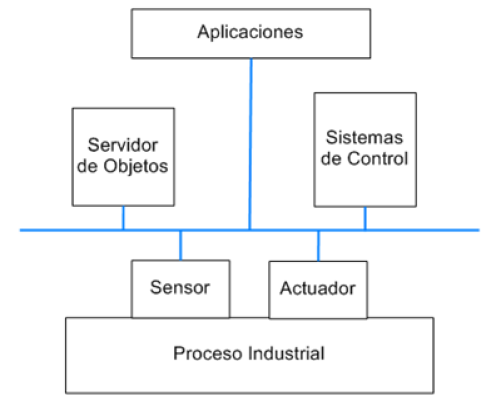

Fig. 1. Esquema de funcionamiento de un sensor.

El monitoreo de variables se lleva a cabo mediante el uso de sensores. Un sensor es un dispositivo que responde a algunas propiedades de tipo eléctrico, mecánico, térmico, magnético, químico, etc., generando una señal eléctrica que puede ser susceptible de medición. Normalmente, las señales obtenidas a partir de un sensor son de pequeña magnitud y necesitan ser tratadas convenientemente en los aspectos de amplificación y filtrado principalmente (García, 2001).

En la figura 2 se puede apreciar un esquema de funcionamiento.

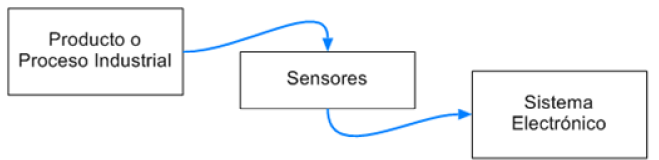

Fig. 2. Esquema de funcionamiento de un sensor.

Una vez convertida en señal eléctrica o electrónica el valor de la variable, se puede digitalizar. Este proceso se puede hacer mediante tarjetas de circuitos electrónicos, llevando la lectura a un computador mediante puertos USB, serial o identificación basada en radio frecuencia - RFID (Mayné, 2003).

Uno de los problemas asociados a la información que se recibe de los sensores es la interpretación de la información, ya que para decidir que ocurrió un evento, hay que analizar una gran cantidad de datos. Esta representación de información debe pasarse a otras instancias del sistema automatizado que tomarán una decisión respecto al evento observado.

Este problema es abordado desde diversos enfoques, como el de la ventana de observaciones (Sarrate, 2002), y otro, es la integración basada en ontologías, que corresponde al enfoque utilizado en el presente trabajo para la representación de la ocurrencia de eventos. 


\section{SISTEMAS MULTI-AGENTE}

La automatización industrial implica que varias unidades de producción cumplan con su misión de control, y que además puedan comunicarse y negociar con otras unidades de producción, de manera que un proceso industrial puede estar distribuido física y lógicamente. Una entidad adecuada para gestionar información en un entorno distribuido son los agentes (Giret, 2005), entre cuyas características se tiene la cooperación, comunicación, adaptabilidad y naturaleza distribuida.

\subsection{Agentes y sistemas multi-agente}

La definición más general de un agente consiste en toda entidad que observa su ambiente, razona de acuerdo a lo observado y actúa en función de unas metas (Russell \& Norvig, 2005). Otras definiciones establecen que un agente es un sistema computacional que se sitúa en algún entorno y es capaz de actuar de forma autónoma para alcanzar sus objetivos de diseño (Wooldridge \& Jennings, 1998). Existen definiciones operacionales en el sentido de que un agente es una entidad que percibe y actúa sobre un entorno a través de sensores y responder según su función en el mismo entorno a través de efectores, asumiendo que cada agente puede percibir sus propias acciones y aprender de la experiencia para definir su comportamiento (Russell \& Norvig, 2005).

Uno de los campos de aplicación de la tecnología de agentes se relaciona con la automatización de procesos y monitoreo de eventos en redes industriales, debido a su naturaleza distribuida, la cual se obtiene cuando los programas que implementan los agentes se crean en varias máquinas diferentes en una red, y se comunican entre sí para desarrollar actividades de manera colaborativa. De esta manera se llega a la definición de sistema multi-agente, donde un sistema dinámico puede contener entidades que deciden las acciones a ejecutar (Ferber \& Muller, 1999). Puesto que un proceso industrial puede modelarse como un sistema dinámico, entonces también se le pueden incorporar los agentes.

Como ejemplo reciente de su aplicación se encuentra una ontología para sistemas holónicos de producción (Checa \& Rojas, 2014). Otros campos de aplicación se encuentran en la gestión de procesos de negocio (Bonfante \& Castillo, 2014), y en el monitoreo de redes (Herrera \& Parra, 2013).

\subsection{Comportamiento de un agente}

Uno de los aspectos más complejos es el de especificar el comportamiento de un agente, donde un enfoque basado en lógica y ciclos de conducta (Kowalski, 2005) es el más adecuado para introducir en sistemas automatizados. Un agente no tiene conocimiento inherente a la actividad que está desarrollando. Corresponde al diseñador agregar las metas de alto nivel, las creencias, y unas reglas que relacionen hechos observados con reacciones. $\mathrm{La}$ especificación de su comportamiento puede establecerse con varios tipos de reglas condición-acción, detección de eventos, derivar sub-metas, ejecutar acciones, confirmar acciones, comunicaciones de y para otros agentes (Parra, 2009). La figura 3 esquematiza un sistema multi-agente para la automatización de procesos. Como puede observarse, existe un repositorio de reglas que especifican su comportamiento. Aunque uno de los inconvenientes es la manera en que va a representarse. Por tal motivo se recurren a las ontologías.

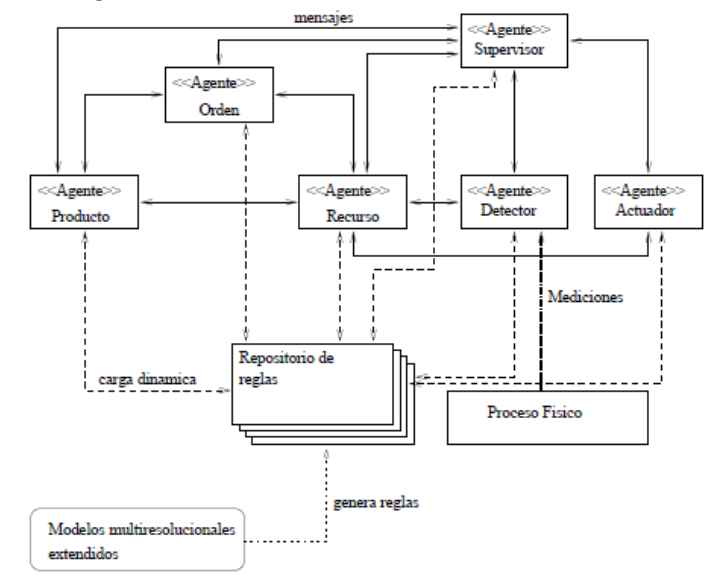

Fig. 3. Esquema de sistema multi-agente en automatización. (Parra, 2009).

\subsection{Ontologías}

Para que los datos que se toman del proceso industrial sean entendibles por múltiples aplicaciones es necesaria la interoperabilidad semántica, la cual necesita de un análisis del contenido. Esto se traduce en la necesidad de especificaciones formales y explícitas de los modelos del dominio que define los términos empleados y sus relaciones. Es precisamente a esos modelos formales a los que se denominan ontologías. En el área de Inteligencia Artificial la ontología se refiere a un esquema de representación constituido por un vocabulario específico empleado para describir un sistema, más un conjunto de supuestos explícitos relacionados con el significado 
atribuido al vocabulario. Este conjunto de supuestos tiene normalmente forma de lógica de primer orden, donde las palabras del vocabulario aparecen como nombres de predicados unitarios o binarios, llamados respectivamente conceptos y relaciones. De esta manera, se describen los conceptos y relaciones para un conjunto de agentes.

\section{DISEÑO DE LA ONTOLOGÍA}

La identificación de los diferentes términos, se realizó a partir del trabajo de tesis de pregrado (Hernández, 2013), basada a su vez del estudio relacionado acerca de la función de los sensores de temperatura y nivel en dos procesos de ejemplo: secado de café y almacenamiento de leche cruda respectivamente.

En este sistema la ontología cubre toda la información sobre la gestión del monitoreo de sensores de los dos procesos elegidos para la toma de decisiones, y cuyos conceptos, acciones y predicados de los agentes se esquematizan en la figura 4 .

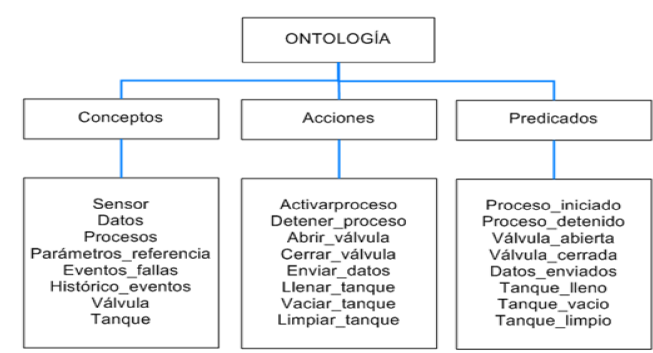

Fig. 4. Ontología diseñada para gestionar el monitoreo de sensores de temperatura y nivel. Fuente: (Hernández, 2013).

\subsection{Conceptos utilizados en la ontología}

A partir del dominio de conocimiento asociado al monitoreo de variables en un proceso industrial, se extrajeron los siguientes conceptos con sus correspondientes atributos:

Sensor: Con este concepto se cubre toda la información necesaria para la identificación del sensor. Los atributos necesarios son:

- descripcion: es el detalle del sensor

- id_sensor: es la identificación del sensor

- estimulo_a_medir: identificación de la variable a medir (nivel o temperatura).
Datos: Este concepto describe la información que los sensores suministran y es analizada por el agente. Los atributos asociados son:

- fecha: fecha en la que el dato es enviado

- hora: hora del dato

- dato: dato enviado por el sensor

- idat_sensor: identificación del sensor que envía los datos

- dat_proceso: identificación del proceso asociado al dato

Procesos: Concepto que relaciona el proceso con los sensores asociados. Los atributos son:

- $\quad$ nom_proceso: nombre del proceso (secar café, almacenar leche)

- id_proceso: identificación del proceso

- id_sensor_proceso: identificación del sensor asociado al proceso

- des_procesos: Descripción del proceso

Parámetros_referencia: El concepto describe la información relacionada a los parámetros de referencia de cada proceso para el correcto funcionamiento. Sus atributos son:

- sensor_parametro_referencia: identifica el sensor asociado al parámetro.

- valor_referencia: son los valores que tiene como referencia el proceso.

- clasificación: la clasificación de los valores (ejm. Por encima, por debajo, normal, alto)

Evento_fallas: Este concepto tiene como objetivo representar una recomendación que se debe realizar ante la ocurrencia de cualquiera de estos eventos o fallas que se pueden presentar en estos procesos. Las subclases y atributos necesarios son:

- descripción_evento: descripción del evento o falla.

- id_evento: identificación del evento o falla.

- nombre_evento: nombre del evento.

- motivo: motivo por el cual se pudo haber presentado el evento o falla.

- id_proceso_evento: identificación del proceso al que está asociado el evento.

- valores_evento: identifica los parámetros de referencia que tiene ese evento.

- recomendación_accion: contiene la acción que se tiene que realizar. 
Histórico_eventos: Este concepto permite relacionar los eventos que han ocurrido en el proceso asociado a determinado sensor. Los atributos son:

- evento_historico: identificación del evento.

- dato_historico_evento: datos del sensor con los que se activó la alarma.

Válvula: Este concepto permite relacionar las válvulas que poseen los procesos las cuales van a ser cerradas o abiertas dependiendo del escenario que se presente.

Los atributos son:

- id_valvula: identificación de la válvula.

- nom_valvula: nombre de la válvula.

- des_valvula: descripción de la válvula.

- id_proceso_valvula: identificación del proceso al que esta asociado la válvula.

- estado_valvula: identifica si la válvula se encuentra sucia o limpia.

Tanque: Este concepto permite relacionar las válvulas que poseen los procesos las cuales van a ser cerradas o abiertas dependiendo del escenario que se presente. Los atributos son:

- id_tanque: identificación del tanque.

- nombre_tanque: nombre del tanque.

- des_tanque: descripción del tanque.

- id_sensor_tanque: identificación del sensor al que está asociado el tanque.

- estado_tanque: estado en el que se encuentra el tanque (Limpio o sucio).

- capacidad_tanque: Capacidad de almacenamiento del tanque.

\subsection{Acciones}

- Activar_proceso: se utiliza esta acción para iniciar el proceso.

- Detener_proceso: se utiliza esta acción para detener el proceso ya sea por falla o por culminación del mismo.

- Abrir_válvula: se utiliza para abrir la válvula asociada a un proceso.

- Cerrar_válvula: se utiliza para cerrar la válvula del proceso asociado.

- Enviar_datos: se utiliza para enviar datos.

- Llenar_tanque: se utiliza para llenar el tanque.

- Vaciar_tanque: se utiliza para vaciar el tanque.

- Limpiar_tanque: se utiliza para limpiar el tanque.

\subsection{Predicados}

- Proceso_iniciado: estado de inicio.

- Proceso_detenido: estado de detención.

- Válvula_abierta: estado de válvula abierta.

- Válvula_cerrada: estado de válvula cerrada.

- Datos_enviados: estado datos enviados.

- Tanque_lleno: estado de tanque lleno.

- Tanque_vacío: estado de tanque vacío.

- Tanque_limpio: estado de tanque limpio

\section{IMPLEMENTACIÓN}

La ontología se implementó por medio de la clase Protégé, de la Universidad de Stanford. El sistema multiagente se implementó mediante la plataforma Jade. La cual se utilizó debido a su entorno gráfico, a la estandarización de comunicación entre agentes utilizando el protocolo estándar FIPA-ACL (véase http://www.fipa.org/specs/fipa00061/index.html), у a su entorno distribuido.

Los agentes que conforman el sistema multi-agente son:

- AgenteMedición: Este agente funciona como una interfaz para el acceso a la información que generan los sensores. Este agente es instanciado para cada sensor. Envía mensaje al agente Controlador con los datos del sensor.

- AgenteControlador: Es el encargado de procesar la información y de tomar las decisiones. Se instancia una sola vez. Analiza los datos recibidos para decidir si se activa una alarma, enviando un mensaje al agente de actuación con la acción a realizar.

- AgenteActuación: Este agente se encarga de ejecutar las decisiones tomadas por el agente controlador, traduce estas decisiones en acciones específicas sobre su entorno. Este agente es instanciado para actuador. Envía mensaje al agente controlador indicando si realizó o no la acción.

La secuencia del paso de mensajes del sistema multi-agente de manera general se ve reflejada en la figura 5 . 


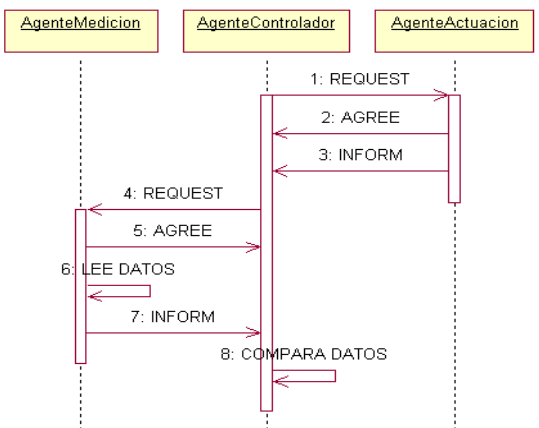

Fig. 5. Diagrama de secuencia de paso de mensajes entre agentes.

\section{APLICACIÓN AL PROCESO DE SECADO DE CAFÉ}

En el mercado mundial del café, la calidad del grano y su consistencia, son factores que influyen en la calidad del grano. En la mayoría de los cultivos en Colombia se seca el café en forma natural o artificial dependiendo del área cultivada y de la capacidad económica del productor. El secado de forma natural, que consiste en extender el grano en patios para que el sol los seque, tarda entre 8 a 15 días efectivos. A causa del clima muchas zonas cafeteras no son adecuadas para secar café al sol debido a la humedad del ambiente o bajas temperaturas, y el café húmedo tiene alto riesgo de ser atacado por hongos o ser contaminado con micotoxinas, causando que el grano pierda su calidad. El café debe ser secado inmediatamente se ha cosechado, esto con el fin de eliminar lo más pronto posible la humedad del grano hasta llegar al $12 \%$ bh para evitar olores y sabores indeseables. El proceso de secado se da cuando se presenta transferencia de calor y masa, como son la humedad interna y el líquido evaporado de las superficies húmedas del grano. En este proceso es importante mantener una temperatura constante entre los 50 y $55^{\circ} \mathrm{C}$ y no exceder de $60^{\circ} \mathrm{C}$ para asegurar una buena calidad, esto conforme a la profundidad de la capa de café, para no deteriorar el grano.

\subsection{Simulación de la cámara secadora}

La simulación se enfoca en el control de la temperatura dentro de la cámara secadora, debido a que el porcentaje de humedad del grano debe ser reducido hasta niveles seguros para el almacenamiento (12\%) y en tiempo real el proceso toma horas para poder lograrlo, solo se simularan tres escenarios en los que se puede seguir el comportamiento que presenta el sensor de temperatura.
La cámara secadora tendrá como objetivo controlar la temperatura en la cámara de secado del café y está compuesta por:

- Un sensor de temperatura.

- Una válvula de control.

- Tanque de GLP.

- Cámara de secado.

- Calentador de aire.

El sensor de temperatura se encarga de medir la temperatura en el interior de la cámara de secado, la fuente de combustible para la producción de calor para el secado es un secador por GLP (Gas Licuado de Petróleo) el cual genera una determinada cantidad de calor, la válvula de control abre y cierra el paso del gas para el encendido del quemador, el calentador de aire el cual funciona de manera similar al termostato.

En la figura 6 se puede apreciar el esquema de funcionamiento de la cámara de secado de café.

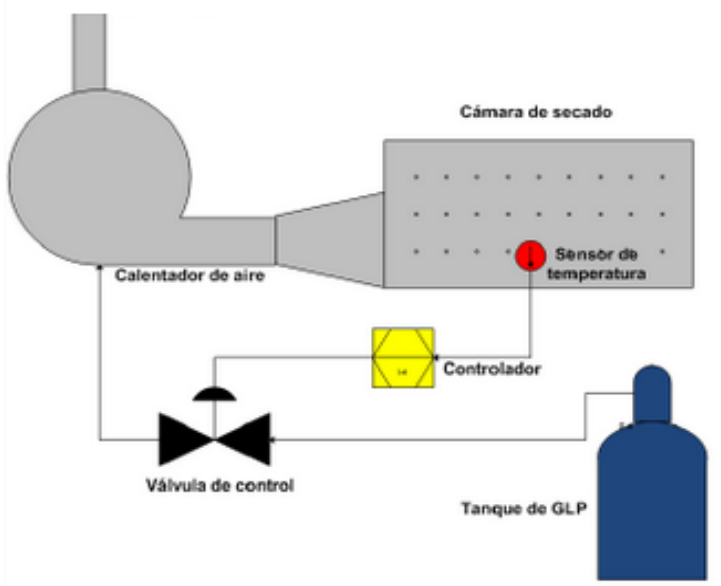

Fig. 6. Funcionamiento del proceso de secado de café.

En la cámara de secado, la temperatura de secado deberá producir el calentamiento del aire hasta una temperatura tal que coincida con el valor requerido.

Se debe mantener la temperatura del aire de secado igual a la temperatura requerida. Para lograrlo, se debe regular el flujo de GLP a través de una electroválvula, hacia un quemador la cuál detiene el flujo de aire en el momento en que el interior de la cámara llega a la temperatura programada.

El sensor del dispositivo de temperatura mide la temperatura del aire que ingresa a la cámara. 


\subsection{Supuestos del modelo}

Las siguientes suposiciones se tienen en cuenta para el modelo:

a) El flujo de aire caliente, a la entrada de la cámara de secado, es constante.

b) La temperatura del aire a la salida del quemador es igual a la temperatura del aire a la entrada de la cámara de secado.

c) La distribución del aire caliente en la cámara de secado es uniforme.

d) La temperatura se mantiene entre los $50 \mathrm{y}$ $55^{\circ} \mathrm{C}$

Se establecieron tres tipos de escenarios que podrían ocurrir en el proceso del secado considerando que la temperatura es inestable debido a otros factores en el sistema (temperatura ambiente, nivel de GLP, humedad inicial del grano, caudal de flujo de salida del gas que es proporcional a la apertura de la llave del regulador o válvula del tanque, tipo de gas GLP, calor liberado en la combustión que es directamente proporcional a la tasa de pérdida de masa y a las características del calentador):

a) Temperatura por debajo de lo establecido: si el sensor detecta que la temperatura está por debajo de lo establecido, el calentador debe comenzar a calentar, los elementos calefactores del interior de la caja del calentador se calientan hasta que el sensor del detecte que el aire que ingresa a la cámara está en el punto de referencia ingresado por el usuario.

b) Temperatura por encima de lo establecido: si el sensor detecta que la temperatura está subiendo más de lo establecido, se debe detener la entrada del flujo de aire a la cámara secadora cerrando la válvula y generando alarma.

c) La temperatura se mantiene estable: el sensor detecta que la temperatura se mantiene dentro de los 50 a $55^{\circ} \mathrm{C}$, el flujo de aire se detiene hasta que la temperatura descienda a $50^{\circ} \mathrm{C}$.

\subsection{Características del sensor de temperatura}

La temperatura es uno de los factores determinantes en el secado de granos, por lo que se requiere de un sensor que proporcione esta información en forma eficaz y cuyos límites de funcionamiento se encuentren entre la temperatura ambiente y la máxima de operación.
El sensor termopar es un sensor sencillo y uno de los más usados en las industrias para determinar la temperatura de un proceso, está constituido por la unión de dos metales distintos que producen un voltaje en función de la diferencia de temperatura entre uno de los extremos denominado punto caliente y el otro conocido como unión fría, este tipo de sensor tiene una determinación puntual y una respuesta rápida a las variaciones de temperatura y tiene un amplio rango de medición. El sistema de adquisición de datos a simular está basado en una placa de adquisición de datos de Nacional Instruments, NI USB-9211 .

Una vez ejecutado el modelo en Java, lo que ocurre es que la variable temperatura empieza a cambiar de acuerdo a las ecuaciones de transferencia de calor, cuando la válvula está apagada, y a ello se le agrega la ecuación de calor debido al efecto del calentador, cuando la válvula se enciende. Dado que diversos agentes están gestionando sensores, controladores y actuadores, la figura 7 muestra el esquema del paso de mensajes entre ellos, más con el agente que lleva a cabo el modelo de simulación, lo que demuestra que efectivamente los agentes tienen en cuenta esta ontología para acordar el control de un proceso.

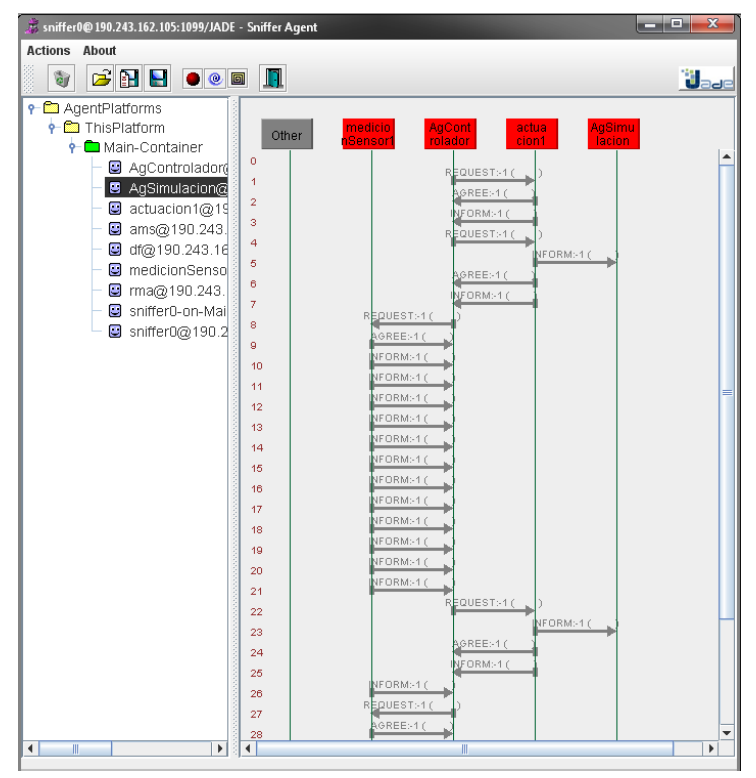

Fig. 7. Paso de mensajes entre agentes que controlan el proceso.

Como resultado de la interacción de los agentes, la temperatura del proceso se mantiene dentro del rango establecido la mayor parte del tiempo.

Como método para analizar los resultados se evaluó la salida del modelo de dos maneras: la consistencia 
del modelo numérico, comparando los valores obtenidos de las variables asociadas al modelo (temperatura, humedad, flujo de salida de gas, entre otros) con aquellos valores esperados obtenidos de una solución analítica. Por otro lado, se hizo un chequeo de los eventos de comunicación entre los agentes, el paso de mensajes entre ellos como evidencia de coordinación, y también se confrontaban si los cambios en el comportamiento de la variable estaban asociados a acciones de los agentes. También se pudo revisar el contenido de los mensajes de los agentes, y revisar si se ajustaban a la ontología propuesta.

\section{CONCLUSIONES}

A partir del trabajo de grado (Hernández, 2013), se entrega un diseño e implementación de una Ontología que mejora el intercambio de información de un sistema multiagente para el monitoreo de sensores y detección de eventos que puedan ocurrir durante la simulación de un proceso simulado, y se demostró que funciona en un entorno multiagente denominado Jade. Los resultados de las simulaciones evidenciaron que la representación de conceptos facilitó que los agentes compartieran información resultando en un logro de sus metas: controlar el proceso que se estaba simulando.

Se pudo constatar como las ontologías pueden ser integradas con un entorno multi-agente basado en java, al aprovechar el mecanismo que da soporte a las ontologías para la realización de la transformación de mensajes ACL y programas de fácil manipulación (codificación y decodificación), permitiendo al lenguaje de contenido realizar la traducción a un String según las reglas sintácticas del lenguaje y por el otro lado la ontología realiza la validación semántica del mensaje.

\section{REFERENCIAS}

Bonfante, M. \& Castillo, A. (2014). Integración de sistema multi-agente, ontologías y procesos de negocio como marco tecnológico de la estrategia "gobierno en línea". Revista Colombiana de Tecnologías de Avanzada (RCTA). Vol. 1, No. 23.

Chacón E., De Sarrazín G. (2004). Automatización de sistemas de producción, Reporte Técnico, Universidad de Los Andes, Venezuela.

Checa, D. \& Rojas, O. (2014). Ontología para los sistemas holónicos de manufactura basados en la unidad de producción. Revista Colombiana de Tecnologías Avanzadas. Vol. 1, No. 23.

Ferber J. \& Muller, J. (1996). Influences and reactions: a model for situated multi-agent systems. ICMAS'96.

García, E. (2001). Automatización de Procesos Industriales. Universidad Politécnica de Valencia. Alfaomega. México.

Giret, A. (2005). ANEMONA, una metodología multi-agente para sistemas holónicos de fabricación, $\mathrm{PhD}$ tesis Universidad Politécnica de Valencia.

Hernández, A. (2013). Diseño de una ontología para agentes que monitorean mediciones de sensores, Tesis de grado, Universidad de Pamplona.

Herrera, J., Parra, C. (2013). Aplicación de los sistemas de detección de intrusos y la tecnología de agentes en el monitoreo inteligente de redes de datos. Revista Colombiana de Tecnologías de Avanzada, Vol. 7, No. 22.

Kowalski, R. (2006). The logical way to be artificially intelligent, Computational logic in multi-agent systems. Springer-Verlag.

Mayné, J. (2003). Sensores acondicionadores y procesadores de señal, Reporte técnico Silica - Avnet, Madrid.

Parra, C. Colina, E. Chacón E. (2008). Design Framework for intelligent supervision of industrial processes, WSEAS Transactions on Systems, Issue 7, Vol. 7.

Parra, C. (2009). Modelado y simulación del control supervisorio para sistemas holónicos de producción continua, Tesis doctoral, Universidad de Los Andes, Venezuela.

Quintero, L. (2010). Un modelo de control inteligente para sistemas de manufactura basado en los paradigmas holónico y multiagente. Tesis de maestría, Universidad Nacional de Colombia, Medellín.

Russell, S. Norvig, P. (2005). Inteligencia artificial, un enfoque moderno, Ed. Pearson, segunda edición, Madrid.

Sarrate, R. (2002). Supervisió intelligent de processos dinamics basada en esdeveniments, PhD. Tesis, Valencia.

Wooldridge, M \& Jennings N. (1998). Agents Technology, Ed. Academic Press, segunda Edición, Londres. 